\title{
Correction to: Efficacy and safety of an innovative prolonged-release combination drug in patients with distal renal tubular acidosis: an open-label comparative trial versus standard of care treatments
}

\author{
Aurélia Bertholet-Thomas ${ }^{1}$ - Catherine Guittet ${ }^{2} \cdot$ Maria A. Manso-Silván ${ }^{2} \cdot$ Arnaud Castang $^{2} \cdot$ Véronique Baudouin ${ }^{3}$. \\ Mathilde Cailliez ${ }^{4}$. Massimo Di Maio ${ }^{5}$. Olivia Gillion-Boyer ${ }^{6} \cdot$ Emilija Golubovic $^{7}$. Jérôme Harambat ${ }^{8}$. \\ Alexandre Klein ${ }^{9} \cdot$ Bertrand Knebelmann $^{10}$. François Nobili ${ }^{11}$. Robert Novo ${ }^{12}$ • Ludmila Podracka $^{13}$. \\ Gwenaëlle Roussey-Kesler ${ }^{14}$. Christos Stylianou ${ }^{15}$. Luc-André Granier ${ }^{2}$
}

Published online: 28 September 2020

(C) IPNA 2020

Correction to: Pediatr Nephrol. https://doi.org/10.1007/s00467-020-04693-2

The published version of the article unfortunately contained a mistake. In several occurrences, the citations to references [11] and [13] were mistakenly cited as [12] and [14] respectively. These errors were introduced during the revision stage. The authors apologize for this mistake. The original article was corrected.

Publisher's note Springer Nature remains neutral with regard to jurisdictional claims in published maps and institutional affiliations.

The online version of the original article can be found at https://oi.org/ 10.1007/s00467-020-04693-2

Aurélia Bertholet-Thomas

aurelia.bertholet-thomas@chu-lyon.fr

1 Centre de Référence des Maladies Rénales Rares - Néphrogones Hôpital Femme Mère Enfant - Filière ORKiD, Hospices Civils de Lyon, Bron, France

2 Advicenne, Nîmes, France

3 Service de Néphrologie Pédiatrique, Hôpital Robert Debré, Paris, France

4 Service de Pédiatrie Multidisciplinaire, Hôpital de la Timone, AP-HM, Marseille, France

5 Service de Réanimation Néonatale et Néonatologie, CHU de Nîmes, Nîmes, France

6 Service de Néphrologie Pédiatrique, Centre de Référence des, Maladies Rénales Héréditaires de l'Enfant et de l'Adulte, (MARHEA), Institut Imagine, Hôpital Necker-Enfants Malades, Université de Paris, Paris, France
7 Klinički Centar Niš, Klinika za dečije interne bolesti, Odeljenje za nefrologiju, Niš, Serbia

8 Service de Pédiatrie, CHU de Bordeaux, Hôpital Pellegrin-Enfants, Bordeaux, France

9 Service de Néphrologie, Pôle DIACOR, Hôpitaux Civils de Colmar, Colmar, France

10 Service de Néphrologie adultes Hôpital Necker, Paris, France

11 Service de Pédiatrie 2, Hôpital Jean Minjoz, CHU de Besançon, Besançon, France

12 Service de Néphrologie Pédiatrique, Hôpital Jeanne de Flandre, CHRU de Lille, Lille, France

13 Department of Pediatrics, National Institute of Children's Health, Bratislava, Slovakia

14 Unité de Néphrologie et Hémodialyse Pédiatrique, Clinique Médicale Pédiatrique Hôpital Mère-Enfant, CHU de Nantes, Nantes, France

15 ClinBay Ltd, Limassol, Cyprus 